\title{
Confidential enquiry into maternal deaths in the Netherlands, 2006-2018: a retrospective cohort study
}

\author{
Athanasios Kallianidis ${ }^{1}$, Joke Schutte ${ }^{2}$, Louise Schuringa ${ }^{1}$, Ingrid Beenakkers ${ }^{3}$, Kitty \\ Bloemenkamp ${ }^{3}$, Babette Braams-Lisman ${ }^{4}$, Jerome Cornette ${ }^{5}$, Simone Kuppens ${ }^{6}$, Anna \\ Rietveld ${ }^{7}$, Timme Schaap ${ }^{3}$, Jelle Stekelenburg ${ }^{8}$, Joost Zwart ${ }^{9}$, and Thomas van den Akker ${ }^{1}$ \\ ${ }^{1} \mathrm{LUMC}$ \\ ${ }^{2}$ Isala Hospitals \\ ${ }^{3} \mathrm{UMC}$ Utrecht \\ ${ }^{4}$ Tergooi \\ ${ }^{5}$ Erasmus MC \\ ${ }^{6}$ Catharina Ziekenhuis \\ ${ }^{7}$ Amsterdam University Medical Centres \\ ${ }^{8} \mathrm{UMCG}$ \\ ${ }^{9}$ Deventer Hospital
}

January 26, 2021

\begin{abstract}
Objective: To calculate maternal mortality ratio (MMR) for 2006-2018 in the Netherlands and compare with $1993-2005$. Describe women's and obstetric characteristics, causes of death and improvable factors. Design: Prospective cohort study. Setting: Nationwide. Population: 2,304,271 livebirths. Methods: Analysis of all maternal deaths between January 1st, 2006, and December, 31st, 2018 as reported to and audited by the national Audit Committee Maternal Mortality and Morbidity. Main outcome measures: MMR, causes of death, improvable factors. Results: Overall MMR was 6.2 per 100,000 livebirths, a decrease from 12.1 in 1993-2005 (Odds Ratio (OR) 0.5, 95\%CI 0.4-0.6). Women with non-Western ethnic background had a slightly increased MMR compared to Dutch women (MMR 6.5 vs 5.0, OR 1.3, 95\% CI 0.9-1.9), and was particularly increased among women with a background from Surinam/Dutch Antilles (MMR 14.7 OR 2.9, 95\%CI 1.6 - 5.3). Half of all women had an uncomplicated medical history $(79 / 161,49.1 \%)$. Of 172 pregnancy-related deaths within one year postpartum, $103(60 \%)$ had a direct and $69(40 \%)$ an indirect cause. Leading causes within 42 days postpartum were cardiac disease $(\mathrm{n}=21,14.8 \%)$, hypertensive disorders $(n=20,14.1 \%)$ and thrombosis $(n=19,13.4 \%)$. For deaths up to one year postpartum, suicide was the third commonest cause $(n=20,11.6 \%)$. Improvable factors in care were identified in $76(47.5 \%)$ of all deaths. Conclusions: Maternal mortality halved in 2006-2018 compared to 1993-2005. Unlike before, cardiac disease outnumbered hypertensive disorders as main cause of death. Women with a background from Surinam/Dutch Antilles had a threefold higher risk of death compared to Dutch women.
\end{abstract}

\section{Hosted file}

Maternal Mortality in the Netherlands 2006 2018_final version.pdf available at https: //authorea.com/users/391810/articles/505814-confidential-enquiry-into-maternal-deathsin-the-netherlands-2006-2018-a-retrospective-cohort-study 